\title{
Remodeling of the tumor/tumor microenvironment ecosystem during KRAS G12C inhibitor clinical resistance in lung cancer
}

\author{
Tadashi Manabe ${ }^{1,2,3}$ and Trever G. Bivona ${ }^{1,2,3}$ \\ 'Department of Medicine, 'Department of Cellular and Molecular Pharmacology, and ${ }^{3}$ Helen Diller Family Comprehensive Cancer Center, UCSF, San Francisco, California, USA.
}

\begin{abstract}
KRAS C12C inhibitors such as sotorasib and adagrasib are often effective in KRAS C12C-driven non-small cell lung cancer (NSCLC) patients. However, acquired resistance limits long-term patient survival. In this issue of the $J C I$, Tsai et al. present a comprehensive genetic analysis of multiple tumors with acquired sotorasib resistance obtained through an autopsy of a patient with KRAS G12C-mutant NSCLC. This analysis of pre- and posttreatment tumors uncovered cancer cell-intrinsic and -extrinsic features of resistance, including reactivation of KRAS-mediated signaling, reprogramming of metabolism, epithelial-mesenchymal transition, and tumor microenvironment changes. This elegant study demonstrates the multifaceted nature of KRAS G12C inhibitor clinical resistance and potential avenues to overcome resistance.
\end{abstract}

\section{KRAS inhibitors in lung cancer} Currently, 1 in 2 to 3 people will develop cancer, and among them, lung cancer remains the leading cause of cancer mortality. Substantial breakthrough discoveries, including the identification of lung cancer-specific oncogenic drivers (e.g., EGFR mutations, EML4-ALK fusion genes) and the development of molecular inhibitors of these pathogenic factors, have improved outcomes for patients with advanced-stage lung cancer. However, frequent oncogenic driver mutations that have remained undruggable until recently include activating mutations in the gene encoding the small GTPase KRAS. Hotspot mutations that predominately occur in codons 12 and 13 result in defective KRAS GTPase activity, thus enhancing the abundance of the active GTP-bound state in cells to promote can- cer cell proliferation and growth via multiple downstream effector pathways such as the RAF/MEK/ERK (MAPK) pathway.

In recent years, a substantial advance occurred through the identification and development of covalent allosteric inhibitors especially targeting KRAS G12C (1-4), a mutation that comprises $41 \%$ of KRAS nonsmall cell lung cancer (NSCLC) (5). One drug, sotorasib (AMG510), inhibits reactivation by nucleotide exchange, thereby better confining KRAS to an inactive GDPbound state (1). A subgroup of patients with NSCLC that underwent a phase I trial of this drug showed a $32.2 \%$ response rate and had 6.3 months of progression-free survival (6). Based on these results, sotorasib was granted accelerated approval by the US Food and Drug Administration in May of 2021 for adult patients with advanced KRAS G12C-mutant NSCLC who received

Delated Article: https://doi.org/10.1172/JCl155523

Conflict of interest: TCB is an advisor to Revolution Medicines, Relay, Rain, Engine, and Scorpion, and is a cofounder of Granule Therapeutics and receives research funding from Revolution Medicines, Kinnate, Strategia, and Verastem. Copyright: $\odot$ 2022, Manabe et al. This is an open access article published under the terms of the Creative Commons Attribution 4.0 International License.

Reference information: / Clin Invest. 2022;132(4):e156891. https://doi.org/10.1172/JCl156891.

at least one prior systemic therapy. While the development of KRAS inhibitors has been a tremendous success, resistance occurs in almost all patients and limits long-term patient survival. A better understanding of the mechanisms of resistance to KRAS inhibitors is paramount to maximize the potential of these emerging agents and improve clinical outcomes.

A variety of resistance mechanisms have been reported in preclinical studies of KRAS G12C inhibitors (2). Additionally, some mechanisms of clinical acquired resistance to KRAS G12C inhibitors have also been reported (6-8). One challenge that often arises in studies using clinical samples is ensuring ideal controls. For instance, preand posttreatment pairs and comparisons with normal tissue are desirable but often difficult to achieve in clinical practice.

\section{Mechanisms of clinical resistance to KRAS G12C inhibitors}

In an elegant study published in this issue of the JCI, Tsai et al. (9) used paired preand post-sotorasib-treatment tissue samples and corresponding normal tissues to elucidate acquired mechanisms of clinical resistance to KRAS G12C inhibitors via comprehensive genetic analyses. The specimens used in this study were obtained from a patient with advancedstage KRAS G12C lung adenocarcinoma treated with sotorasib for 17 weeks, with an initial response followed by acquired resistance (Figure 1). After succumbing to drug-resistant tumor progression, this patient underwent a rapid autopsy through which abundant tumor specimens and controls were obtained: 4 tumor tissues before treatment, 13 tumor tissues after therapy, and 8 nonadjacent normal tissues.

First, the authors performed transcriptome analysis of lymph node tumors and found that a total of 950 genes were differentially expressed after sotorasib treat- 


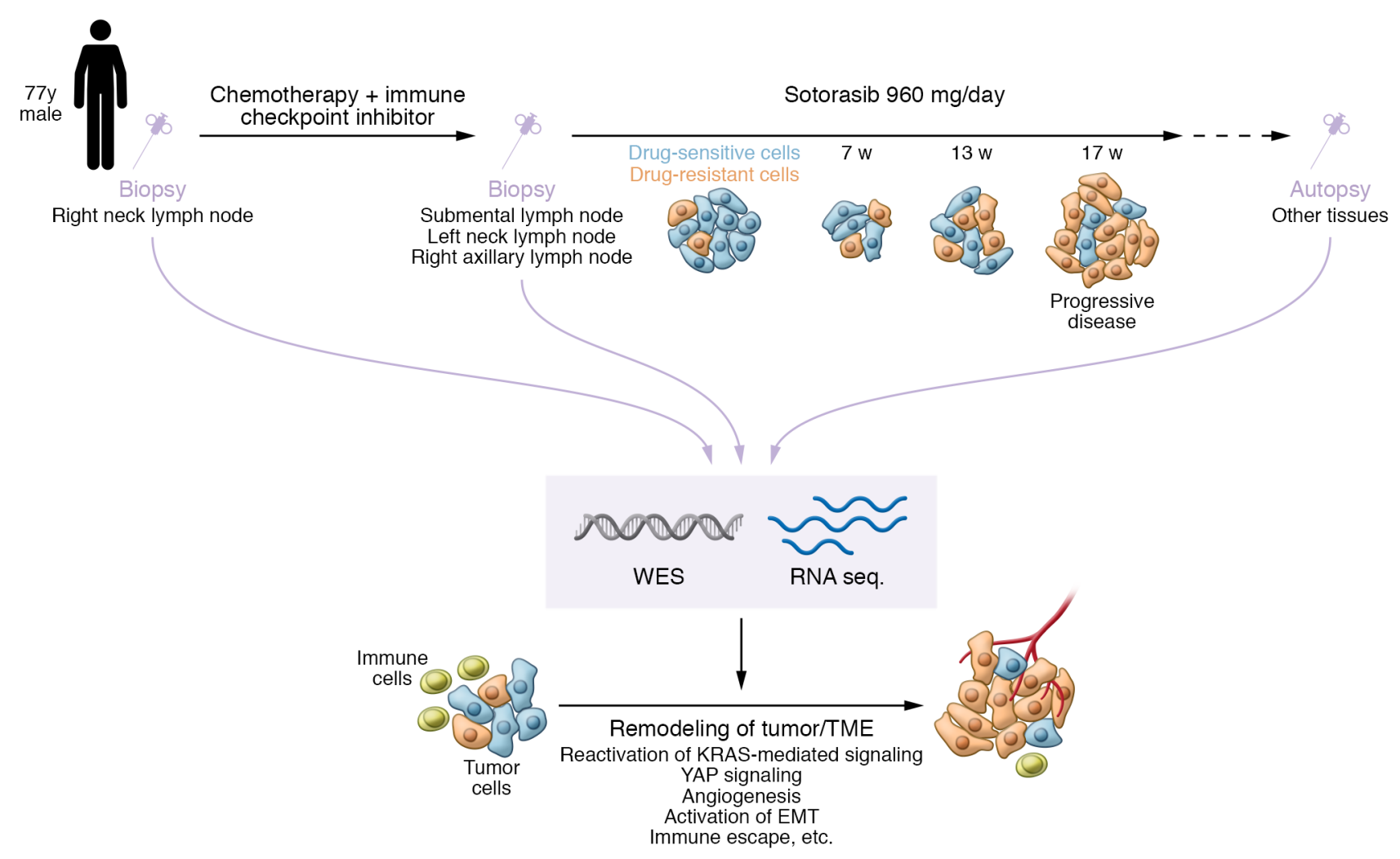

Figure 1. Schema showing the approach and main findings of Tsai et al. (9). Biopsy samples from a patient with KRAS G12C lung adenocarcinoma were taken before and after treatment with the KRAS G12C inhibitor, sotorasib. The patient was treated for 17 weeks and showed an initial response before developing acquired resistance. Whole-exome sequencing (WES) and RNA sequencing analyses of tumor and normal tissue samples showed KRAS-mediated signaling activation, YAP signaling, EMT activation, metabolic reprogramming, and TME changes that included coagulation, angiogenesis, and immune escape pathways.

ment. Compared with pretreatment samples, activation of MAPK pathway, AKT, and mTOR signaling was present in almost all samples after sotorasib treatment. This result was consistent with previous reports describing that reactivation of MAPK and/ or PI3K/AKT/mTOR pathways induce varying degrees of resistance $(2,10)$. However, unlike several previous reports investigating the mechanism of KRAS-inhibitor resistance using sequencing techniques $(7,8,11)$, the authors did not find any additional mutations that reactivate KRAS or MAPK signaling. Whole-exome sequencing also showed a reduced KRAS G12C mutant allele frequency in most samples after treatment, suggesting that other factors maintain reactivation of the MAPK and/or PI3K/AKT/mTOR pathways. Activation of YAP signaling as a compensatory pathway supporting drug resistance was also present in the resistant tumors, consistent with preclinical studies (12).

In the 9 hallmark gene sets analyzed, 2 cell cycle gene sets $\left(G_{2} / M\right.$ checkpoint and E2F target) were decreased, suggest- ing abnormal cell proliferation. The other 7 gene sets showed upregulation of a wide variety of pathways, including activation of hedgehog, NOTCH, WNT/ $\beta$-catenin signaling, epithelial-mesenchymal transition (EMT), and tumor angiogenesis.

Phylogenetic analysis performed in Tsai et al. (9) revealed the process of inferred clonal evolution across metastatic lesions in different anatomic sites. Specifically, the pretreatment submental lymph node metastases had evidence of a common subclonal origin with the 6 posttreatment distant metastases, suggesting it seeded these distant posttreatment resistant tumors. Additional phylogenetic analysis of adjacent periportal lymph nodes showed a divergent inferred clonal origin. This result suggests that even adjacent metastatic lesions may arise from different clonal populations and have distinct resistance mechanisms, consistent with real-world clinical experience where treatment response can vary according to metastatic site.
Tsai et al. (9) also evaluated immunogenomic features of the tumors to understand how the immune microenvironment of tumors differs upon treatment exposure and resistance. The findings showed that multiple immune gene signatures associated with $\mathrm{T}$ and $\mathrm{B}$ cell function and activation were reduced in the posttreatment samples, resulting in an immunologically cold state.

\section{Conclusions and clinical implications}

This important study by Tsai and colleagues illuminates the multifaceted mechanisms of acquired resistance to direct inhibition of KRAS G12C, including the reactivation of KRAS-mediated signaling, YAP signaling, the activation of EMT, metabolic reprogramming, and diverse changes in the tumor microenvironment (TME), including coagulation, angiogenesis, and immune escape pathways (ref. 9 and Figure 1). This study highlights mechanisms that warrant 
validation in other patients with KRAS G12C NSCLC and in preclinical models, as showing generalizability and causation are critical areas for future investigation. It is also important to define the extent to which the mechanisms of resistance to the different KRAS inhibitors, in clinical use or development, are shared or distinct. Overall, Tsai et al. (9) show the utility of obtaining and assessing clinical specimens as pre- and posttreatment pairs, comprehensively across the tumor/ TME ecosystem, and characterizing both DNA and RNA levels, as well as normal tissue controls, to identify tumor-specific resistance alterations. Comprehensive tissue-based molecular analyses of tumors with clinical resistance to KRAS G12C inhibitors, coupled with liquid biopsy assessment and preclinical mechanistic studies, should yield a full evolutionary map by which tumors develop resistance. This evaluative process is essential to lay the groundwork for testing mechanism-based therapeutic strategies to thwart resistance and improve clinical outcomes for patients with KRAS G12C-driven NSCLC and potentially other KRAS-driven tumor types.

\section{Acknowledgments}

TM receives funding from the Uehara Memorial Foundation. TGB receives funding from the National Cancer Institute of the $\mathrm{NIH}$ (grants R01CA204302, U54CA224081, R01CA169338, R01CA211052, and R01CA231300).

Address correspondence to: Trever G. Bivona, 600 16th Street, Genentech Hall, N232, San Francisco, California 94158, USA. Phone: 415.502.0237; Email: trever. bivona@ucsf.edu.

1. Canon J, et al. The clinical KRAS(G12C) inhibitor AMG 510 drives anti-tumour immunity. Nature. 2019;575(7781):217-223.

2. Hallin J, et al. The KRAS(G12C) inhibitor MRTX849 provides insight toward therapeutic susceptibility of KRAS-mutant cancers in mouse models and patients. Cancer Discov. 2020;10(1):54-71.

3. Janes MR, et al. Targeting KRAS mutant cancers with a covalent G12C-specific inhibitor. Cell. 2018;172(3):578-589.
4. Lito P, et al. Allele-specific inhibitors inactivate mutant KRAS G12C by a trapping mechanism. Science. 2016;351(6273):604-608.

5. Biernacka A, et al. The potential utility of re-mining results of somatic mutation testing: KRAS status in lung adenocarcinoma. Cancer Genet. 2016;209(5):195-198.

6. Hong DS, et al. KRAS(G12C) inhibition with sotorasib in advanced solid tumors. N Engl J Med. 2020;383(13):1207-1217.

7. Awad MM, et al. Acquired resistance to KRAS(G12C) inhibition in cancer. $N$ Engl J Med. 2021;384(25):2382-2393.

8. Zhao Y, et al. Diverse alterations associated with resistance to KRAS(G12C) inhibition. Nature. 2021;599(7886):679-683.

9. Tsai YS, et al. Rapid idiosyncratic mechanisms of clinical resistance to KRAS G12C inhibition. JClin Invest. 2022;132(4):e155523.

10. Misale S, et al. KRAS G12C NSCLC models are sensitive to direct targeting of KRAS in combination with PI3K inhibition. Clin Cancer Res. 2019;25(2):796-807.

11. Tanaka N, et al. Clinical acquired resistance to KRAS(G12C) inhibition through a novel KRAS switch-II pocket mutation and polyclonal alterations converging on RAS-MAPK reactivation. Cancer Discov. 2021;11(8):1913-1922.

12. Kapoor A, et al. Yap1 activation enables bypass of oncogenic Kras addiction in pancreatic cancer. Cell. 2014;158(1):185-197. 